\title{
Decoherent Histories Analysis of Models without Time
}

\author{
J. J. Halliwell \\ Theory Group, Blackett Laboratory, Imperial College, London SW7 2BZ, UK \\ Received on 11 December, 2004
}

\begin{abstract}
The decoherent histories approach is a particularly useful approach to quantum theory especially when time enters in a non-trivial way, or indeed, when there is no physical time coordinate at all, as is the case in quantum cosmology. Here, attempts to apply the decoherent histories approach to quantum cosmology are described.
\end{abstract}

\section{INTRODUCTION}

The subject of quantum cosmology was born in the 1960's with the work of DeWitt, Misner, Wheeler and others [1-3]. The subject took off in the early 1980's with Hartle and Hawking's seminal paper, The Wave Function of the Universe [4]. This development was very pertinent since the appearance of the inflationary universe scenario at about that time underscored the urgency to acquire an understanding of cosmological initial conditions. The new ingredients of quantum cosmology, compared to its older 1960s version, were two-fold. First, there was the use of Euclidean path integral methods which had grown out of their very successful application to black holes physics. Secondly, and perhaps more importantly, there was the Hartle-Hawking "no-boundary" proposal. This gave, perhaps for the first time, a genuinely quantum gravitational proposal which implied cosmological initial conditions. In this proposal, wave functions satisfying the WheelerDeWitt equation

$$
\mathcal{H} \Psi\left[h_{i j}\right]=0
$$

are given by a sum over histories expression of the form

$$
\Psi\left[h_{i j}\right]=\int_{C} \mathcal{D} g_{\mu \nu} \exp \left(-I\left[g_{\mu v}\right]\right)
$$

where the sum is over closed four-geometries whose only boundary is the three-surface on which the three-metric $h_{i j}$ is specified. (Note also that the functional integral is taken to be over a complex contour $C$ [5]).

There were two questions of particular interest in these early days. Firstly, what sort of picture can quantum gravity give about the initial singularity? And secondly, what initial conditions does the theory imply for classical cosmological solutions? In simple, somewhat heuristic models, the answer to the first question is that the initial singularity is replaced by some kind of quantum tunneling situation. That is, the classical singularity can (at least in some models) become a classically forbidden region. The second question can be answered by showing how quantum cosmology produces, in a quasiclassical limit, an approximate probability distribution on the space of classical cosmological solutions.

The most pressing questions in those days were to do with cosmological issues: Does the no-boundary proposal predict initial conditions which produce inflationary solutions and subsequent structure formation $[6,7]$ ? The interpretation associated with these models was heuristic [7-10]. For example, one considered the Klein-Gordon flux

$$
J=i\left(\Psi^{*} \nabla \Psi-\Psi \nabla \Psi^{*}\right)
$$

associated with the wave functions of the WKB type,

$$
\Psi=C e^{i S}
$$

These heuristic methods appeared to be adequate for the questions considered at the time.

Now, however, far more is known about the foundations and interpretation of quantum theory. Moreover, there has also been a considerable amount of activity in the experimental tests of foundational ideas. As a result of this, the foundations of quantum theory is now more in the mainstream of physics, when previously it was regarded as the domain of the philosophers. Perhaps as a result of this, there has been a secondary wave of much slower activity in quantum cosmology, that aims to look more deeply into a whole variety of mathematical and conceptual issues related to the application of quantum theory to the universe as a whole. In particular, it is now reasonable to ask, how can the heuristic ideas used earlier be derived or reconciled with a properly defined interpretational structure for quantum theory of models described by a Wheeler-DeWitt equation? For example, in standard quantum theory, probabilities generally have the form

$$
P=\operatorname{Tr}(P \rho)
$$

where $\rho$ is the density operator, $P$ is a projection operator and the trace is over a complete set of states (and so assumes a Hilbert space of states with a suitably defined inner product). How can the predictions of quantum cosmology be reconciled with a formula of this type?

Related to these issues is the notorious problem of time [10-12]. The Wheeler-DeWitt equation, in simple minisuperspace models, has the form,

$$
H \Psi=\left(-\nabla^{2}+U\right) \Psi=0
$$

where $\nabla^{2}$ is a d'Alembertian type operator. The equation is of the form of a Klein-Gordon equation in a general curved spacetime background with a space-time dependent mass term. There is no single variable to play the role of time, nor is there the possibility of splitting the solutions into positive and negative frequency. In the usual Klein-Gordon equation one then resorts to second quantization, but the Wheeler-DeWitt equation is in some sense already the second quantized theory, so one has to face the issue of the lack of a time coordinate more squarely.

In this contribution to the conference, I will describe how the decoherent histories approach may be used to provide a quantization of simple minisuperspace models, perhaps avoiding some of the serious difficulties outlined above, and agreeing with the heuristic methods used earlier on. 
I begin by making two simple observations about the Wheeler-DeWitt equation that are generally relevant to the discussion. The first is that, at least classical speaking, the constraint equation,

$$
H=f^{a b} p_{a} p_{b}+V=0
$$

is related to reparametrization invariance. (This is the leftover of four-dimensional diffeomorphism invariance after the restriction to minisuperspace). In keeping with Dirac's general ideas about quantizing constrained systems, we therefore look for observables- quantities which commute with the constraint. There has been a certain amount of debate about this issue in the context of general relativity, but this will not affect us here [13-15].

Second, as observed by Barbour [16, 17], by analogy with Mott's 1929 calculation of tracks in a cloud chamber [18], there is a natural association between the Wheeler-DeWitt equation and the emergence of classical trajectories. Mott asked why the outgoing spherical wave associated with alpha decay produced a straight line track in a cloud chamber. By considering the Hamiltonian of the decaying atom interacting with ionizing particles in the cloud chamber he showed that the ionized particles with high probability lie along a straight line. Interestingly, and as Barbour observed, Mott actually solved the time-independent Schrödinger equation,

$$
H \Psi=E \Psi
$$

rather than the time-dependent one, to obtain the tracks. This is therefore an interesting analogy for the Wheeler-DeWitt equation where, in the quasiclassical limit one expects to obtain emergent classical trajectories. (In fact, a model of the Wheeler-DeWitt equation including explicit detectors, and exhibiting these features has been constructed [19]. Some related approaches to timeless models are Refs.[20-22].)

With these preliminaries out of the way, we now focus on the following simple question: Suppose we have an $n$ dimensional configuration space with coordinates $\mathbf{x}=\left(x_{1}, x_{2}, \cdots x_{n}\right)$, and suppose the wave function of the system is in an eigenstate of the Hamiltonian,

$$
H \Psi\left(x_{1}, x_{2}, \cdots x_{n}\right)=E \Psi\left(x_{1}, x_{2}, \cdots x_{n}\right)
$$

What is the probability of finding the system in a series of regions of configuration space $\Delta_{1}, \Delta_{2}, \cdots \Delta_{N}$ without reference to time?

This question will form the main focus of the rest of this paper. We first consider the classical case, and then the decoherent histories analysis.

\section{THE CLASSICAL CASE}

We begin by considering the classical case which contains many almost all the key features of the problem. (We follow the treatment of Ref.[23] quite closely). For simplicity we will concentrate on the case of a single region of configuration space $\Delta$
We will consider a classical system described by a $2 n$ dimensional phase space, with coordinates and momenta $(\mathbf{x}, \mathbf{p})=\left(x_{k}, p_{k}\right)$, and Hamiltonian

$$
H=\frac{\mathbf{p}^{2}}{2 M}+V(\mathbf{x})
$$

More generally, we are interested in a system for which the kinetic part of the Hamiltonian has the form $g^{k j}(\mathbf{x}) p_{k} p_{j}$, where $g^{k j}(\mathbf{x})$ is an inverse metric of hyperbolic signature. Most minisuperspace models in quantum cosmology have a Hamiltonian of this form. However, the focus of this paper is the timelessness of the system, and the form of the configuration space metric turns out to be unimportant. So for simplicity, we will concentrate on the form Eq.(10).

We assume that there is a classical phase space distribution function $w(\mathbf{p}, \mathbf{x})$, which is normalized according to

$$
\int d^{n} p d^{n} x w(\mathbf{p}, \mathbf{x})=1
$$

and obeys the evolution equation

$$
\frac{\partial w}{\partial t}=\sum_{k}\left(-\frac{p_{k}}{M} \frac{\partial w}{\partial x_{k}}+\frac{\partial V}{\partial x_{k}} \frac{\partial w}{\partial p_{k}}\right)=\{H, w\}
$$

where $\{$,$\} denotes the Poisson bracket. The interesting case$ is that in which $w$ is the classical analogue of an energy eigenstate, in which case $\partial w / \partial t=0$, so the evolution equation is simply

$$
\{H, w\}=0
$$

It follows that

$$
w\left(\mathbf{p}^{c l}(t), \mathbf{x}^{c l}(t)\right)=w(\mathbf{p}(0), \mathbf{x}(0))
$$

where $\mathbf{p}^{c l}(t), \mathbf{x}^{c l}(t)$ are the classical solutions with initial data $\mathbf{p}(0), \mathbf{x}(0)$, so $w$ is constant along the classical orbits. (The normalization of $w$ then becomes an issue if the classical orbits are infinite. This is addressed in Ref.[23]).

Given a set of classical solutions $\left(\mathbf{p}^{c l}(t), \mathbf{x}^{c l}(t)\right)$, and a phase space distribution function $w$, we are interested in the probability that a classical solution will pass through a region $\Delta$ of configuration space. We construct this as follows. First of all we introduce the characteristic function $f_{\Delta}(\mathbf{x})$ of the region $\Delta$, equal to 1 for $\mathbf{x}$ in $\Delta$ and zero otherwise. To see whether the classical trajectory $\mathbf{x}^{c l}(t)$ intersects this region, consider the phase space function

$$
A\left(\mathbf{x}, \mathbf{p}_{0}, \mathbf{x}_{0}\right)=\int_{-\infty}^{\infty} d t \delta^{(n)}\left(\mathbf{x}-\mathbf{x}^{c l}(t)\right)
$$

(In the case of periodic classical orbits, the range of $t$ is taken to be equal to the period). This function is positive for points $\mathbf{x}$ on the classical trajectory labeled by $\mathbf{p}_{0}, \mathbf{x}_{0}$ and zero otherwise. Hence intersection of the classical trajectory with the region $\Delta$ means,

$$
\int d^{n} \mathbf{x} f_{\Delta}(\mathbf{x}) \int_{-\infty}^{\infty} d t \delta^{(n)}\left(\mathbf{x}-\mathbf{x}^{c l}(t)\right)>0
$$


Or equivalently, that

$$
\int_{-\infty}^{\infty} d t f_{\Delta}\left(\mathbf{x}^{c l}(t)\right)>0
$$

This quantity is essentially the amount of parameter time the trajectory spends in the region $\Delta$. We may now write down the probability for a classical trajectory entering the region $\Delta$. It is,

$$
p_{\Delta}=\int d^{n} p_{0} d^{n} x_{0} w\left(\mathbf{p}_{0}, \mathbf{x}_{0}\right) \theta\left(\int_{-\infty}^{\infty} d t f_{\Delta}\left(\mathbf{x}^{c l}(t)\right)-\varepsilon\right)
$$

In this construction, $\varepsilon$ is a small positive number that is eventually sent to zero, and is included to avoid possible ambiguities in the $\theta$-function at zero argument. The $\theta$-function ensures that the phase space integral is over all initial data whose corresponding classical trajectories spend a time greater than $\varepsilon$ in the region $\Delta$.

The classical solution $\mathbf{x}^{c l}(t)$ depends on some fiducial initial coordinates and momenta, $\mathbf{x}_{0}$ and $\mathbf{p}_{0}$, say. In the case of a free particle, for example,

$$
\mathbf{x}^{c l}(t)=\mathbf{x}_{0}+\frac{\mathbf{p}_{0} t}{M}
$$

The construction is independent of the choice of fiducial initial points. If we shift $\mathbf{x}_{0}, \mathbf{p}_{0}$ along the classical trajectories, the measure, phase space distribution function $w$ and the $\theta$ function are all invariant. Hence the integral over $\mathbf{x}_{0}, \mathbf{p}_{0}$ is effectively a sum over classical trajectories. The shift along the classical trajectories may also be thought of as a reparametrization, and the quantity (18) is in fact a reparametrizationinvariant expression of the notion of a classical trajectory. This means that the probability (18) has the form of a phase space overlap of the "state" with a reparametrization-invariant operator.

It is useful also to write this result in a different form, which will be more relevant to the results we get in the quantum theory case. In the quantum theory, we generally deal with propagation between fixed points in configuration space, rather than with phase space point. Therefore, in the free particle case, consider the change of variables from $\mathbf{x}_{0}, \mathbf{p}_{0}$ to $\mathbf{x}_{0}, \mathbf{x}_{f}$, where

$$
\mathbf{x}_{f}=\mathbf{x}_{0}+\frac{\mathbf{p}_{0}}{M} \tau
$$

Hence $\mathbf{x}_{f}$ is the position after evolution for starting from $\mathbf{x}_{0}$ for parameter time $\tau$. The probability then becomes

$$
p_{\Delta}=\frac{M}{\tau} \int d^{n} x_{f} d^{n} x_{0} w\left(\mathbf{p}_{0}, \mathbf{x}_{0}\right) \theta\left(\int_{-\infty}^{\infty} d t f_{\Delta}\left(\mathbf{x}_{0}^{f}(t)\right)-\varepsilon\right)
$$

where $\mathbf{p}_{0}=M\left(\mathbf{x}_{f}-\mathbf{x}_{0}\right) / \tau$ and

$$
\mathbf{x}_{0}^{f}(t)=\mathbf{x}_{0}+\frac{\left(\mathbf{x}_{f}-\mathbf{x}_{0}\right)}{\tau} t
$$

The parameter $\tau$ may in fact be scaled out of the whole expression, hence the probability is independent of it.

The result now has the form of an integral over "initial" and "final" points, analogous to similar results in quantum theory.
The result is again essentially a sum over classical trajectories with the trajectories now labeled by any pair of points $\mathbf{x}_{0}$, $\mathbf{x}_{f}$ along the trajectories, and is invariant under shifting $\mathbf{x}_{0}$ or $\mathbf{x}_{f}$ along those trajectories. Naively, one might have thought that the restriction to paths that pass through $\Delta$ is imposed by summing over all finite length classical paths which intersect $\Delta$ as they go from the "initial" point $\mathbf{x}_{0}$ to "final" point $\mathbf{x}_{f}$, that is, $\Delta$ lies between the initial and final points. This is also what one might naively expect in the quantum theory version. However, one can see from the above construction that the correct answer is in fact to sum over all classical paths (which can be of infinite length) passing through $\mathbf{x}_{0}$ and $\mathbf{x}_{f}$ that intersect $\Delta$ at any point along the entire trajectory, even if $\Delta$ does not lie between the two points. This feature is related to the reparametrization invariance of the system.

The above point turns out to be quite crucial to what follows in the rest of this paper, so it is worth saying it in an alternative form. Loosely speaking, the statement is that only the entire classical path respects the reparametrization invariance associated with the constraint equation. A section of the classical path does not. This may be expressed more precisely in terms of the function $A\left(\mathbf{x}, \mathbf{p}_{0}, \mathbf{x}_{0}\right)$ introduced in Eq.(15). This function is concentrated on the entire classical trajectory, and is zero when $\mathbf{x}$ is not on the trajectory. It is easy to see that it has vanishing Poisson bracket with the Hamiltonian $H=H\left(\mathbf{p}_{0}, \mathbf{x}_{0}\right)$, since we have

$$
\begin{aligned}
\left\{H, A\left(\mathbf{x}, \mathbf{p}_{0}, \mathbf{x}_{0}\right)\right\} & =\int_{-\infty}^{\infty} d t\left\{H, \delta^{(n)}\left(\mathbf{x}-\mathbf{x}^{c l}(t)\right)\right\} \\
& =-\int_{-\infty}^{\infty} d t \frac{d}{d t} \delta^{(n)}\left(\mathbf{x}-\mathbf{x}^{c l}(t)\right) \\
& =0
\end{aligned}
$$

This is the precise sense in which the entire trajectory is reparametrization invariant, and the phase space function $A$ may be regarded as an observable - a quantity which commutes with the constraint $H[14,15]$. By way of comparison, consider a second phase space function similarly defined, but on only a finite section of trajectory,

$$
B\left(\mathbf{x}, \mathbf{p}_{0}, \mathbf{x}_{0}\right)=\int_{0}^{\tau} d t \delta^{(n)}\left(\mathbf{x}-\mathbf{x}^{c l}(t)\right)
$$

It is easily seen that

$$
\left\{H, B\left(\mathbf{x}, \mathbf{p}_{0}, \mathbf{x}_{0}\right)\right\}=-\delta\left(\mathbf{x}-\mathbf{x}^{c l}(\tau)\right)+\delta\left(\mathbf{x}-\mathbf{x}^{c l}(0)\right)
$$

Hence $B$ "almost" commutes with $H$, failing only at the end points, and it is in this sense that a finite section of trajectory does not fully respect reparametrization invariance.

A third version of the classical result is also useful. It is of interest to obtain an expression for the probability for intersecting an $(n-1)$-dimensional surface $\Sigma$. Since the result (18) involves the parameter time spent in a finite volume region $\Delta$ it does not apply immediately. However, suppose that the set of trajectories contained in the probability distribution $w$ intersect the $(n-1)$-dimensional surface $\Sigma$ only once. Then we may consider a finite volume region $\Delta$ obtained by thickening $\Sigma$ along the direction of the classical flow. If this thickening 
is by a small (positive) parameter time $\Delta t$, then the quantity appearing in the $\theta$-function in (18) is

$$
\int d t \int_{\Delta} d^{n} x \delta^{(n)}\left(\mathbf{x}-\mathbf{x}^{c l}(t)\right)=\Delta t I\left[\Sigma, \mathbf{x}^{c l}(t)\right]
$$

where

$$
I\left[\Sigma, \mathbf{x}^{c l}(t)\right]=\int d t \int_{\Sigma} d^{n-1} x \mathbf{n} \cdot \frac{d \mathbf{x}^{c l}(t)}{d t} \delta^{(n)}\left(\mathbf{x}-\mathbf{x}^{c l}(t)\right)
$$

Here, $\mathbf{n}$ is the normal to $\Sigma$, and we suppose that the normal is chosen so that $\mathbf{n} \cdot d \mathbf{x}^{c l} / d t$ is positive. The quantity $I\left[\Sigma, \mathbf{x}^{c l}(t)\right]$, in a more general context, is the intersection number of the curve $\mathbf{x}^{c l}(t)$ with the surface $\Sigma$, and takes the value 0 for no intersections, or \pm 1 (depending on whether there is an even or odd number of intersections). In this case we have assumed that the trajectories intersect at most once, hence $I=0$ or 1 . We then have

$$
\theta(\Delta t I-\varepsilon)=\theta\left(I-\varepsilon^{\prime}\right)=I
$$

(where $\varepsilon=\Delta t \varepsilon^{\prime}$ ) and the probability for intersecting $\Sigma$ may be written

$$
\begin{aligned}
p_{\Sigma} & =\int d t \int d^{n} p_{0} d^{n} x_{0} w\left(\mathbf{p}_{0}, \mathbf{x}_{0}\right) \\
& \times \int_{\Sigma} d^{n-1} x \mathbf{n} \cdot \frac{d \mathbf{x}^{c l}(t)}{d t} \delta^{(n)}\left(\mathbf{x}-\mathbf{x}^{c l}(t)\right)
\end{aligned}
$$

At each $t$, we may perform a change of variables from $\mathbf{p}, \mathbf{x}$ to new variables $\mathbf{p}^{\prime}=\mathbf{p}^{c l}(t), \mathbf{x}^{\prime}=\mathbf{x}^{c l}(t)$, and using Eq.(14), we obtain the result

$$
p_{\Sigma}=\frac{1}{M} \int d t \int_{\Sigma} d^{n} p^{\prime} d^{n-1} x^{\prime} \mathbf{n} \cdot \mathbf{p}^{\prime} w\left(\mathbf{p}^{\prime}, \mathbf{x}^{\prime}\right)
$$

Finally, the integrand is now in fact independent of $t$, so the $t$ integral leads to an overall factor. (This might be infinite but is regularized as discussed below). We therefore drop the $t$ integral.

This result is relevant for the following reason. In the heuristic "WKB interpretation" of quantum cosmology, one considers WKB solutions to the Wheeler-DeWitt equation of the form

$$
\Psi=C e^{i S}
$$

It is usually asserted that this corresponds to a set of classical trajectories with momentum $\mathbf{p}=\nabla S$, and with a probability of intersecting a surface $\Sigma$ given in terms of the flux of the wave function across the surface [7-9]. As we shall show, from the decoherent histories analysis, the quantum theory gives a probability for crossing a surface $\Sigma$ proportional to Eq.(30) with $w$ replaced by the Wigner function of the quantum theory. The Wigner function of the WKB wave function is, approximately [9],

$$
W(\mathbf{p}, \mathbf{x})=|C(\mathbf{x})|^{2} \delta(\mathbf{p}-\nabla S)
$$

Inserting in Eq.(30), we therefore obtain, up to overall factors, the probability distribution,

$$
p_{\Sigma}=\int_{\Sigma} d^{n-1} x \mathbf{n} \cdot \nabla S|C(\mathbf{x})|^{2}
$$

We therefore have agreement with the usual heuristic analysis.

\section{THE DECOHERENT HISTORIES APPROACH TO QUANTUM THEORY}

Our aim is to analyze the quantum case using the decoherent histories approach to quantum theory [24]. We first give a very brief review of the formalism.

In non-relativistic quantum mechanics, quantum histories are represented by so-called class operators $C_{\underline{\alpha}}$, which are given by time-ordered sequences of projection operators

$$
C_{\underline{\alpha}}=P_{\alpha_{n}}\left(t_{n}\right) \cdots P_{\alpha_{1}}\left(t_{1}\right)
$$

(or more generally, by sums of terms of this form), where $\underline{\alpha}$ denotes the string of alternatives $\alpha_{1}, \alpha_{2} \cdots \alpha_{n}$, and $P_{\alpha_{k}}(t)$ are projection operators in the Heisenberg picture. The central object of interest is then the decoherence functional,

$$
D\left(\underline{\alpha}, \underline{\alpha^{\prime}}\right)=\operatorname{Tr}\left(C_{\underline{\alpha}} \rho C_{\underline{\alpha}^{\prime}}^{\dagger}\right)
$$

Intuitively, the decoherence functional is a measure of the interference between pairs of histories $\underline{\alpha}, \underline{\alpha^{\prime}}$. When its real part is zero for $\underline{\alpha} \neq \underline{\alpha^{\prime}}$, we say that the histories are consistent and probabilities

$$
p(\underline{\alpha})=D(\underline{\alpha}, \underline{\alpha})
$$

obeying the usual probability sum rules may be assigned to them. Typical physical mechanisms which produce this situation usually cause both the real and imaginary part of $D\left(\underline{\alpha}, \underline{\alpha}^{\prime}\right)$ to vanish. This condition is usually called decoherence of histories, and is related to the existence of so-called generalized records.

In the non-relativistic case, for histories characterized by projections onto configuration space, a path integral version of the decoherence functional is available, and can be very useful. It has the form,

$$
\begin{aligned}
D\left(\underline{\alpha}, \underline{\alpha}^{\prime}\right) & =\int_{\underline{\alpha}} \mathcal{D} x \int_{\underline{\alpha}^{\prime}} \mathcal{D} y \exp \left(\frac{i}{\hbar} S[x(t)]-\frac{i}{\hbar} S[y(t)]\right) \\
& \times \rho\left(x_{0}, y_{0}\right)
\end{aligned}
$$

where the sum is over pairs of paths $x(t), y(t)$ passing through the pairs of regions $\underline{\alpha}, \underline{\alpha^{\prime}}$. This is equivalent to the form (34), (35), when the histories are strings of projections onto ranges of positions. Eq.(37) is a useful starting point for the generalization to timeless theories.

The power of the decoherent histories approach is that it readily generalizes to a variety of different situations in which time plays a non-trivial role [25-27]. In particular, it may be generalized to the question of interest here, in which the system is in an energy eigenstate and we would like to answer questions that do no refer to time in any way. This generalization requires, however, specification of the inner product used to construct the decoherence functional, and a prescription for the construction of the class operators. We consider each in turn. 


\section{THE INDUCED INNER PRODUCT}

For many situations, and especially for the analogous situation in quantum cosmology, the Hamiltonian has a continuous spectrum so the energy eigenstates are not normalizable in the usual inner product,

$$
\left\langle\Psi_{1} \mid \Psi_{2}\right\rangle=\int d^{n} x \Psi_{1}^{*}(\mathbf{x}) \Psi_{2}(\mathbf{x})
$$

A way to deal with this has been developed, and goes by the name of the induced inner product, or Rieffel induction [28, 29]. Consider the eigenvalue equation

$$
H\left|\Psi_{E \lambda}\right\rangle=E\left|\Psi_{E \lambda}\right\rangle
$$

where $\lambda$ denotes the degeneracy. These eigenstates will typically satisfy

$$
\left\langle\Psi_{E^{\prime} \lambda^{\prime}} \mid \Psi_{E \lambda}\right\rangle=\delta\left(E-E^{\prime}\right) \delta_{\lambda \lambda^{\prime}}
$$

from which it is clear that the inner product diverges when $E=$ $E^{\prime}$. The induced inner product on a set of eigenstates of fixed $E$ is defined, loosely speaking, by discarding the $\delta$-function $\delta\left(E-E^{\prime}\right)$. That is, the induced or physical inner product is then defined by

$$
\left\langle\Psi_{E \lambda^{\prime}} \mid \Psi_{E \lambda}\right\rangle_{p h y s}=\delta_{\lambda \lambda^{\prime}}
$$

This procedure can be defined quite rigorously, and has been discussed at some length in Refs.[28, 29]. We will use it here to construct the decoherence functional. A simple prescription for using it in the decoherence functional is to regularize each propagator and energy eigenstate by using a different energy for each. The final answer will then involve a number of $\delta$ functions in energy, as in (40), which are simply dropped.

Applied to the Klein-Gordon equation in flat space, where solutions may be split into positive and negative frequency parts, $\phi=\phi^{+}+\phi^{-}$, the induced inner product may be expressed in terms of the Klein-Gordon inner product (which is negative on the negative frequency solutions):

$$
(\phi, \phi)_{I}=\left(\phi^{+}, \phi^{+}\right)_{K G}-\left(\phi^{-}, \phi^{-}\right)_{K G}
$$

This is clearly positive definite. That is, the induced inner product effectively changes the sign of the negative frequency solutions to make the overall expression positive definite. Importantly, the induced inner product does not require a split into positive and negative frequency solutions, and so is generally applicable in quantum cosmological models.

Although the induced inner product has been around for a long time (in the Klein-Gordon case it was noted by Henneaux and Teitelboim [30]), it is only comparatively recently that it made an appearance in quantum cosmology.

\section{THE CLASS OPERATORS}

The most important part of the construction of the decoherence functional for our timeless model is the construction of the class operators. On the face of it, a natural prescription for constructing these is the following expression:

$$
\begin{aligned}
C_{\Delta}\left(\mathbf{x}_{f}, \mathbf{x}_{0}\right) & =\int_{-\infty}^{\infty} d \tau e^{-i E \tau} \int \mathcal{D} \mathbf{x}(t) \exp (i S[\mathbf{x}(t)]) \\
& \times \theta\left(\int_{0}^{\tau} d t f_{\Delta}(\mathbf{x}(t))-\varepsilon\right)
\end{aligned}
$$

This expression consists of a functional integral between the initial and final points in fixed parameter time $\tau$ over paths constrained to enter the region $\Delta$ (enforced by the $\theta$-function as in the classical case), followed by a sum over all possible parameter times $\tau$ [31]. In keeping with the general ideas of Dirac quantization, we expect that, in order that the reparametrization invariance of the theory is fully respected, a properly constructed class operator should be annihilated by the constraint,

$$
H C_{\Delta}=0
$$

It is straightforward to show that this is indeed the case in the limit that $\Delta$ becomes the entire configuration space. However, as shown in Ref.[23], this is not in fact the case when the region $\Delta$ is finite: one obtains $\delta$-functions on the boundary of $\Delta$ on the right-hand side of Eq.(44).

This is a serious difficulty since it means that reparametrization invariance is in some sense violated. It is due to the fact that, as the end-points move from inside $\Delta$ to outside, the class operator changes discontinuously. This in turn is related to the fact that a projection operator onto the region $\Delta$ does not commute with the constraint equation.

Because of this difficulty, it is necessary to replace the class operator $C_{\Delta}$ with a modified class operator $C_{\Delta}^{\prime}$ which is as much as possible, defined by a sum over paths passing through $\Delta$ but satisfies the constraint equation everywhere [28]. At present, there does not appear to be a universally agreed way to do this, but some suggestions and discussion of this point were given in Refs.[23, 28, 32].

Fortunately, in the semiclassical approximation, it seems clear how to construct the modified propagator. It is

$$
\begin{aligned}
C_{\Delta}^{\prime}\left(\mathbf{x}_{f}, \mathbf{x}_{0}\right) & =\theta\left(\int_{-\infty}^{\infty} d t f_{\Delta}\left(\mathbf{x}_{0}^{f}(t)\right)-\varepsilon\right) \\
& \times P\left(\mathbf{x}_{f}, \mathbf{x}_{0}\right) e^{i A\left(\mathbf{x}_{f}, \mathbf{x}_{0}\right)}
\end{aligned}
$$

Here $P e^{i A}$ is the usual unrestricted semiclassical propagator, so $A\left(\mathbf{x}_{f}, \mathbf{x}_{0}\right)$ is the classical action between initial and final points, and $P$ is a prefactor. The $\theta$-function here is the same as in the (rewritten) classical case Eq.(21) in terms of "initial" and "final" points, where $\mathbf{x}_{0}^{f}(t)$ denotes that classical path from $\mathbf{x}_{0}$ to $\mathbf{x}_{f}$. (This is exactly as in the classical case depicted in Fig.1). Note also that

$$
\nabla A \cdot \nabla \theta\left(\int_{-\infty}^{\infty} d t f_{\Delta}\left(\mathbf{x}_{0}^{f}(t)\right)-\varepsilon\right)=0
$$

as may be shown by shifting the $t$ integration. It follows that the modified class operator is a semiclassical solution to the constraint equation, as required. 
It is important that $t$ is integrated over an infinite range in the quantity inside the $\theta$-function, otherwise the modified class operator would not in fact satisfy the constraint. Recall that the originally defined class operator Eq.(43) contained a similar $\theta$-function, with a finite range of time integration, which one might have been tempted to use in the semiclassical approximation, but this class operator does not in fact satisfy the constraint.

Hence we see that the difference between the modified and original class operators in the semiclassical approximation is the difference between using the entire classical trajectory or using finite segments of it in the $\theta$-functions. We also see that these modified class operators are the correct ones to use in order to be consistent with the discussion of the classical case and Eq.(21). There, we saw that it is appropriate to sum over classical paths intersecting $\Delta$ even if $\Delta$ does not lie on the segment of classical trajectory between $\mathbf{x}_{0}$ and $\mathbf{x}_{f}$. This feature therefore appears to be necessary for the particular type of reparametrization invariance used here. Only the entire trajectory is reparametrization-invariant notion. A finite section of trajectory is not. (See Ref.[33] for a further discussion of reparametrizations in this sort of context).

Given a decoherence mechanism (not discussed here), it may be shown that this modified class operator leads to the expected heuristic results [23]. However, this leaves the question as to what is the exact form, in the full quantum theory, of the class operator that respects the constraints. At the conference I briefly described a possible answer to this problem. The idea is to consider the projection operator $P$ onto the region $\Delta$ (so the quantum version of the window function $f_{\Delta}(\mathbf{x})$ ). Associated with this is the projection $\bar{P}=1-P$, onto the region outside $\Delta$. Then let us ask for the class operator $\bar{C}_{\Delta}$ for trajectories which never enter $\Delta$. The proposal is that this is given by the infinite product over time,

$$
\bar{C}_{\Delta}=\prod_{t} \bar{P}(t)
$$

where $\bar{P}(t)=e^{i H t} \bar{P} e^{-i H t}$. The desired class operator $C_{\Delta}$ is then equal to $1-\bar{C}_{\Delta}$.

The point is that this object (which clearly requires some mathematical work to define) is, at least formally, invariant under reparametrizations, so commmutes with $H$. Furthermore, classically, it is equal to either zero or 1, depending on whether the trajectories do not or do enter the region $\Delta$. Hence is seems a reasonable object to investigate. One can see, at least heuristically, that it agrees with the guessed forms of the modified class operators (at least up to factors of $\delta(H)$, which make no difference in the induced inner product formalism). Work is currently in progress to explore the full consequences of this proposal and will be described in detail elsewhere.

\section{SUMMARY AND DISCUSSION}

The present work shows that it is possible to carry out a decoherent histories quantization of simple quantum cosmological models. This quantization method reproduces, approximately, heuristic methods based on essentially classical ideas. These models have the key property of not possessing a time parameter, but this does not appear to present an insurmountable obstruction to the application of the approach.

Central to both the classical and quantum problems is the notion of an entire trajectory. At the classical level it appears to be the appropriate reparametrization-invariant notion for the construction of interesting probabilities. At the quantum level, the decoherent histories approach appears to handle the problem in a natural way, perhaps because it readily incorporates the notion of trajectory. This approach to quantizing cosmological models in this way is certainly only a first bite at the problem, and a list of further topics and related issues is described at some length in Ref.[23].

\section{ACKNOWLEDGEMENTS}

I am very grateful to Thomas Elze for giving me the chance to participate in this most interesting meeting. I would also like to thank Jim Hartle for many discussions, over a long period of time, on the subject of this paper.
[1] B.S. DeWitt, Phys. Rev. 160, 1113 (1967).

[2] C.W. Misner, in Magic Without Magic: John Archibald Wheeler, a Collection of Essays in Honor of his 60th Birthday, edited by J.Klauder (Freeman, San Francisco,1972).

[3] J.A. Wheeler, in Batelles Rencontres, eds.C.DeWitt and J.A. Wheeler (Benjamin, New York, 1968)

[4] J.B. Hartle and S.W. Hawking, Phys. Rev. 28, 2960 (1983).

[5] J.J. Halliwell and J.B. Hartle, Phys. Rev. D 41, 1815 (1990).

[6] J.J. Halliwell and S.W. Hawking, Phys. Rev. D 31, 1777 (1985).

[7] S.W. Hawking and D.N. Page, Nucl. Phys.B 264, 185 (1986); B 298, 789 (1988).

[8] J.J. Halliwell, in, Proceedings of the 13th International Conference on General Relativity and Gravitation, edited by R.J. Gleiser, C.N. Kozameh, O.M. Moreschi (IOP Publishers, Bris- tol,1992). (Also available as the e-print gr-qc/9208001).

[9] J.J. Halliwell, Phys. Rev. D 36, 3626 (1987).

[10] K. Kuchar, in Conceptual Problems of Quantum Gravity, edited by A. Ashtekar and J. Stachel (Boston, Birkhauser, 1991); and in Proceedings of the 4th Canadian Conference on General Relativty and Relativistic Astrophysics, edited by G. Kunstatter, D.E. Vincent and J.G. Williams (World Scientific, New Jersey, 1992). See also the e-print gr-qc/9304012, Canonical quantum gravity.

[11] J. Butterfield and C.J. Isham, gr-qc/9901024.

[12] C.J. Isham, gr-qc/9210011.

[13] B. DeWitt, in Gravitation: An Introduction to Current Research, edited by L. Witten (John WIley and Sons, New York, 1962). 
[14] D. Marolf, Class. Quant. Grav 12, 1199 (1995).

[15] C. Rovelli, Class. Quant. Grav 8, 297 (1991); 8, 317 (1991).

[16] J. Barbour, Class. Quant. Grav. 11, 2853 (1994).

[17] J. Barbour, Class. Quant. Grav. 11, 2875 (1994).

[18] N.F. Mott, Proc. Roy. Soc A 124, 375 (1929), reprinted in Quantum Theory and Measurement, edited by J. Wheeler and W. Zurek (Princeton University Press, Princeton, New Jersey, 1983). For further discussions of the Mott calculation see also, J.S. Bell, Speakable and Unspeakable in Quantum Mechanics (Cambridge University Press, Cambridge, 1987); A.A. Broyles, Phys. Rev. A 48, 1055 (1993); M. Castagnino and R. Laura, grqc/0006012.

[19] J.J. Halliwell, Phys. Rev. D64, 044008 (2001).

[20] M. Montesinos, C. Rovelli, and T. Thiemann, Phys. Rev. D 60 , 044009 (1999); M. Montesinos, Gen. Rel. Grav. 33, 1 (2001); M. Montesinos and C. Rovelli, Class. Quantum Grav. 18, 555 (2001).

[21] C. Rovelli, Phys. Rev. 42, 2638 (1990).

[22] C. Rovelli, Phys. Rev. 43, 442 (1991).

[23] J.J. Halliwell and J. Thorwart, Phys. Rev. D 65, 104009 (2002).

[24] M. Gell-Mann and J.B. Hartle, in Complexity, Entropy and the Physics of Information, SFI Studies in the Sciences of Complexity, Vol. VIII, W. Zurek (ed.) (Addison Wesley, Reading, 1990); and in Proceedings of the Third International Symposium on the Foundations of Quantum Mechanics in the Light of New Technology, S. Kobayashi, H. Ezawa, Y. Murayama and S. Nomura (eds.) (Physical Society of Japan, Tokyo, 1990); Phys. Rev. D 47, 3345 (1993); R.B. Griffiths, J.Stat.Phys. 36, 219 (1984); Phys. Rev. Lett. 70, 2201 (1993); Am. J. Phys. 55, 11 (1987); R. Omnès, J. Stat. Phys. 53, 893 (1988); 53, 933 (1988); 53, 957 (1988); 57, 357 (1989); 62, 841 (1991); Ann. Phys. 201, 354 (1990); Rev. Mod. Phys. 64, 339 (1992); J.B. Hartle, in
Quantum Cosmology and Baby Universes, S. Coleman, J. Hartle, T. Piran and S. Weinberg (eds.) (World Scientific, Singapore, 1991); J.J. Halliwell, in Fundamental Problems in Quantum Theory, edited by D. Greenberger and A. Zeilinger, Annals of the New York Academy of Sciences, Vol 775, 726 (1994). For further developments in the decoherent histories approach, particularly adpated to the problem of spacetime coarse grainings, see C. Isham, J.Math.Phys. 23, 2157 (1994); C. Isham and N. Linden, J. Math. Phys. 35, 5452 (1994); 36, 5392 (1995).

[25] D. Craig and J.B. Hartle, Phys. Rev. D69, 123525 (2004).

[26] J. Whelan, Phys. Rev. D 50, 6344 (1994).

[27] N. Yamada and S. Takagi, Prog. Theor. Phys. 85, 985 (1991); 86, 599 (1991); 87, 77 (1992); N. Yamada, Sci. Rep. Tôhoku Uni., Series 8, 12, 177 (1992); Phys. Rev. A 54, 182 (1996); J.J. Halliwell and E. Zafiris, Phys. Rev. D 57, 3351 (1998); J.B. Hartle, Phys. Rev. D 44, 3173 (1991); R.J. Micanek and J.B. Hartle, Phys. Rev. A 54, 3795 (1996).

[28] J.B. Hartle and D. Marolf, Phys. Rev. D 56, 6247 (1997).

[29] A. Ashtekar, J. Lewandowski, D. Marolf, J. Mourao, and T. Thiemann, J. Math. Phys. 36, 6456 (1995); A. Higuchi, Class. Quant. Grav 8, 1983 (1991). D. Giulini and D. Marolf, Class. Quant. Grav 16, 2489 (1999); Class. Quant. Grav 16, 2479 (1999). F. Embacher, Hadronic J. 21, 337 (1998); N. Landsmann, J. Geom. Phys. 15, 285 (1995); D. Marolf, grqc/0011112.

[30] M. Henneaux and C. Teitelboim, Ann. Phys. (N.Y. 143127 (1982).

[31] J.B. Hartle, in Proceedings of the 1992 Les Houches School, Gravity and its Quantizations, edited by B.Julia and J. ZinnJustin (Elsevier Science B.V. 1995)

[32] J.J. Halliwell and J. Thorwart, Phys. Rev. D64, 124018 (2001)

[33] J.J. Halliwell and J.B. Hartle, Phys. Rev. D43, 1170 (1991). 